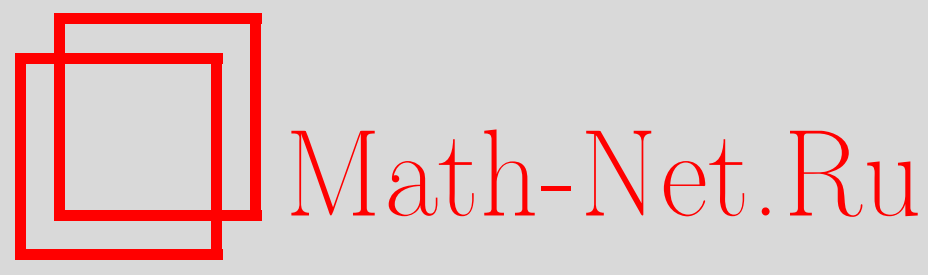

Е. К. Логинов, О лупах Бола, Матем. заметки, 2004, том 75, выпуск 5, 693-701

DOI: https://doi.org/10.4213/mzm65

Использование Общероссийского математического портала Math-Net.Ru подразумевает, что вы прочитали и согласны с пользовательским соглашением http://www.mathnet.ru/rus/agreement

Параметры загрузки:

IP : 54.162 .85 .209

26 апреля 2023 г., 11:02:42

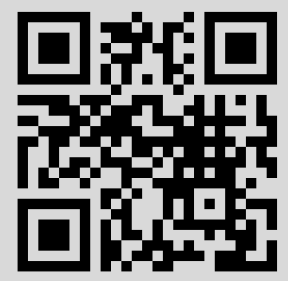


УДК 512

\section{О ЛУПАХ БОЛА}

\section{Е.К. Логинов}

В настоящей статье исследуются лупы Бола и связанные с ними группы. Нами предложен универсальный способ построения лупы Бола, найдены критерии ее простоты и конечности.

Библиография: 5 названий.

1. Напомним, что лупа $S$ назьвается лупой Бола, если

$$
((x y) z) y=x((y z) y)
$$

для всех $x, y, z \in S$. Лупа Бола назьвается лупой Муфанг, если она удовлетворяет тождеству

$$
y(z(y x))=(y(z y)) x .
$$

Тождества Бола (1) и (2) появились в связи с так называемыми условиями замыкания Бола в теории сетей. Пример $x \cdot y=x-y$ на $\mathbb{R}$ показьвает, что оба тождества в квазигруппе независимы и не влекут существования единицы. Хорошо известно (см., например, [1]), что лупа Бола моноассоциативна и обратима справа, причем обратимая слева лупа Бола является лупой Муфанг.

Определим на лупе Бола $S$ композицию $x \cdot y=\left(x y^{-1}\right) x$. Используя очевидное тождество $((x y) x)^{-1}=\left(x^{-1} y^{-1}\right) x^{-1}$, легко видеть, что

$$
\begin{gathered}
x . x=x, \\
x .(x . y)=y, \\
x .(y . z)=(x . y) .(x . z) .
\end{gathered}
$$

Бинарная система $S$, удовлетворяющая тождествам (3)-(5), называется сим.метрическим пространством. Симметрическое пространство с выделенной точкой $e \in S$ на-

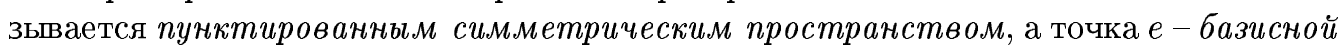
точкой пространства $S$. Изоморфизмы и автоморфизмы симметрических пространств определяются обычньм способом. В случае пунктированных пространств дополнительно требуется, чтобы гомоморфизм переводил базисную точку в базисную. По индукции можно определить степени элемента $x$ (относительно базисной точки $e$ ):

$$
x^{0}=e, \quad x^{1}=x, \quad x^{n+2}=x \cdot\left(e \cdot x^{n}\right), \quad x^{-n}=e \cdot x^{n} .
$$


При этом отображение $x \rightarrow x^{-1}$ будет автоморфизмом симметрического пространства.

Настоящая статья организована следующим образом. В п. 2 мы рассматриваем типичную ситуацию абстрактной теории симметрических пространств: грушу $G$, снабженную инволютивным автоморфизмом, и структуру симметрического пространства на $G$, определенную этой парой. С этим симметрическим пространством естественно связывается некоторое симметрическое пространство $S$ и находятся достаточные условия, при которых естественная бинарная композиция превращает $S$ в лупу Бола (или лупу Муфанг). В п. 3 среди тех груп, которые с помощью этой конструкции приводят к заданной лупе Бола, строится универсальная, из которой все прочие получаются как гомоморфньй образ. Это позволяет дать критерий простоты лупы Бола $S$. В последнем пункте доказывается, что конечность $S$ влечет финитную аппоксимируемость построенной универсальной группы.

2. Пусть $G$ - группа, допускающая инволютивньй автоморфизм $\sigma, I_{\sigma}$ - множество неподвижных точек $\sigma$, и пусть $G_{\sigma}=\left\{x^{-1} x^{\sigma} \mid x \in G\right\}$. Хорошо известно (см. [2]), что множество $G / I_{\sigma}$ правых смежных классов групш $G$ по подгрупше $I_{\sigma}$ является симметрическим пространством относительно произведения

$$
I_{\sigma} x \cdot I_{\sigma} y=I_{\sigma} y^{\sigma}\left(x^{\sigma}\right)^{-1} x
$$

а множество $G_{\sigma}$ - симметрическим пространством относительно произведения

$$
x . y=x y^{-1} x .
$$

При этом отображение $\psi: G / I_{\sigma} \rightarrow G_{\sigma}$, определенное равенством $\psi\left(I_{\sigma} x\right)=x^{-1} x^{\sigma}$, задает изоморфизм $G / I_{\sigma} \simeq G_{\sigma}$ симметрических пространств.

Пусть $N$ - подгруппа в $G$, порожденная множеством $G_{\sigma}$. Предположим, что сушествует гомоморфизм $\varphi: N \rightarrow G$ группы $N$ в группу $G$ такой, что

1) $G=\left\langle x, x^{\sigma} \mid x \in \varphi\left(G_{\sigma}\right)\right\rangle$;

2) $\varphi\left(\varphi(x)^{-1} \varphi(x)^{\sigma}\right)=\varphi(x)$ для всех $x \in G_{\sigma}$;

3) каждый смежньй класс групшы $\varphi(N)$ по $I=I_{\sigma} \cap \varphi(N)$ содержит элементы из $\varphi\left(G_{\sigma}\right)$.

(Здесь и всюду ниже запись $G=\langle M\rangle$ означает, что группа $G$ порождается множеством $M$.) В этом случае групп $G$ будем называть группой с $(\sigma, \varphi)$-свойством или $(\sigma, \varphi)$-группой. Кроме того, всюду ниже символами $N$ и $I$ будем обозначать соответствуюшие подгруппы $(\sigma, \varphi)$-групшы $G$, а символами $H^{\sigma}$ и $H^{\varphi}$ - образы $\sigma(H)$ и $\varphi(H)$ подгрупшы $H$ в $G$.

Обозначим символом $G^{+}$групу $G$, рассматриваемую как пунктированное симметрическое пространство с произведением (6) и базисной точкой 1. Очевидно, что ограничение гомоморфизма $\varphi$ с $N$ на $G_{\sigma}$ индуцирует гомоморфизм $G_{\sigma} \rightarrow G^{+}$пунктированных симметрических пространств. Выберем абстрактное симметричное пространство $S$ изоморфное $\varphi\left(G_{\sigma}\right)$, и пусть $P: S \rightarrow \varphi\left(G_{\sigma}\right)$ - соответствующий изоморфизм. Легко видеть, что для каждого смежного класса $I g$, где $g \in N^{\varphi}$, существует в точности один элемент $x \in S$ такой, что

$$
\operatorname{Ig} \cap \varphi\left(G_{\sigma}\right)=P_{x}
$$


(Здесь и всюду ниже для сокрашения записи мы используем символ $P_{x}$ вместо $P(x)$.) Действительно, если $I P_{x}=I P_{y}$, то $P_{x}^{-1} P_{x}^{\sigma}=P_{y}^{-1} P_{y}^{\sigma}$, и поэтому

$$
P_{x}=\varphi\left(P_{x}^{-1} P_{x}^{\sigma}\right)=\varphi\left(P_{y}^{-1} P_{y}^{\sigma}\right)=P_{y} .
$$

Следовательно, мы можем определить подстановочное представление группы $N^{\varphi}$ на $S$, полагая $x g=z$, если $I P_{x} g=I P_{z}$ для $g \in N^{\varphi}$.

Теорема 1. Если группа $G$ обладает $(\sigma, \varphi)$-свойством, то бинарная компози-

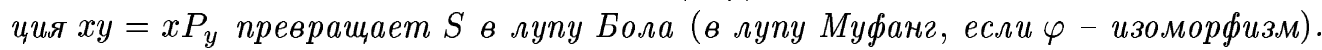

ДокАЗАТЕльство. Фиксируем в пространстве $S$ базисную точку $е$. Поскольку гомоморфизм пунктированных пространств переводит базисную точку в базисную, справедливы равенства $P_{e}=1$ и $P_{x^{-1}}=P_{x}^{-1}$. Поэтому

$$
e x=x=x e \quad \text { и } \quad(y x) x^{-1}=y .
$$

Далее, $P_{x . y^{-1}}=P_{x} P_{y} P_{x}$. Поэтому

$$
x \cdot y^{-1}=(x y) x
$$

и, следовательно,

$$
z((x y) x)=((z x) y) x .
$$

Тем самым доказано, что $S$ - правая лупа Бола с единицей. Покажем, что в лупе $S$ уравнение $a x=b$ имеет единственное решение. Действительно, если решение существует, то оно единственно, поскольку

$$
a^{-1}((a x) a)=x a \quad \text { и } \quad x=\left(a^{-1}(b a)\right) a^{-1} .
$$

С другой стороны,

$$
a\left(\left(a^{-1}(b a)\right) a^{-1}\right)=(b a) a^{-1}=b .
$$

Поэтому решение уравнения $a x=b$ существует. Следовательно, $S$ - лупа Бола.

Пусть $\varphi: N \rightarrow G$-изоморфизм групшы $N$ в группу $G$. Тогда отображение $L: S \rightarrow G^{+}$, определенное равенством $L_{x}=P_{x}^{-1} P_{x}^{\sigma}$, будет изоморфизмом пунктированных симметрических пространств. Поэтому $L_{x^{-1}}=L_{x}^{-1}$ и

$$
\left(P_{x} L_{y}\right)^{-1}\left(P_{x} L_{y}\right)^{\sigma}=L_{\left(y^{-1} x\right) y^{-1}}=P_{\left(y^{-1} x\right) y^{-1}}^{-1} P_{\left(y^{-1} x\right) y^{-1}}^{\sigma} .
$$

Следовательно,

$$
I P_{x} L_{y}=I P_{\left(y^{-1} x\right) y^{-1}}
$$

Тем самьм определено представление $x \rightarrow x L_{y}=\left(y^{-1} x\right) y^{-1}$ группы $N$ на $S$. С другой стороны,

$$
P_{x^{-1}}^{\sigma} P_{x}=L_{x}^{-1}=L_{x^{-1}}=P_{x} P_{x^{-1}}^{\sigma} \quad \text { и } \quad y P_{x} L_{x}=y L_{x} P_{x}
$$

Поэтому

$$
\left(x^{-1}(y x)\right) x^{-1}=\left(\left(x^{-1} y\right) x^{-1}\right) x=x^{-1} y \quad \text { и } \quad x\left(x^{-1} y\right)=x\left(\left(x^{-1}(y x)\right) x^{-1}\right)=y .
$$

Отсюда следует, что лупа Бола $S$ является лупой с обратимостью, а значит, и лупой Муфанг. Теорема доказана. 
СлЕДСТВИЕ 1. Порожсающие әлементы $P_{x} u R_{x}=P_{x^{-1}}^{\sigma}(\sigma, \varphi)$-әруппы $G$ удовлетворяют следующим тождествам:

$$
\begin{gathered}
P_{e}=R_{e}=1 \\
P_{x^{-1}}=P_{x}^{-1}, \quad R_{x^{-1}}=R_{x}^{-1} \\
P_{(x y) x}=P_{x} P_{y} P_{x}, \quad R_{(x y) x}=R_{x} R_{y} R_{x}, \\
P_{x y} P_{y}^{-1} P_{x}^{-1}=R_{x y}^{-1} R_{y} R_{x} .
\end{gathered}
$$

ДоказАтЕльство. Равенства (7)-(9) непосредственно следуют из теоремы 1. Для доказательства равенств (10) достаточно заметить, что

$$
\left(P_{x} P_{y}\right)^{-1}\left(P_{x} P_{y}\right)^{\sigma}=P_{x y}^{-1} P_{x y}^{\sigma}
$$

СЛЕДСТВИЕ 2. Если $G$ - әруппа $c(\sigma, \varphi)$-свойством, mo подәрупnа $I$ в $N^{\varphi}$ noрождается әлементами $P_{x, y}=P_{x} P_{y} P_{x y}^{-1}$, әде $x, y \in S$. В частности, $I=\left\{g \in N^{\varphi} \mid\right.$ $e g=e\}$.

ДокАЗАТЕЛЬСТво. Пусть $H=\left\langle P_{x, y} \mid x, y \in S\right\rangle$ и $K=\left\{g \in N^{\varphi} \mid e g=e\right\}$, где $e$ - единица лупы $S$. Очевидно, что $H \subseteq I \subseteq K$. Покажем, что $K \subseteq H$. Действительно, $N^{\varphi}=\left\langle P_{x} \mid x \in S\right\rangle$. Поэтому всякий элемент из $K$ можно представить в виде слова $W=P_{x_{1}} P_{x_{2}} \ldots P_{x_{n}}$. Если $n=1$, то $W=P_{e} \in H$. Если $n>1$, то $W=P_{x_{1}, x_{2}} P_{x_{1} x_{2}} \ldots P_{x_{n}}$, и утверждение доказьвается очевидной индукцией по длине слова $W$.

Лупу Бола $S=S(G)$ и связанную с ней $(\sigma, \varphi)$-группу $G$ будем называть ассоииированными. Если $H-$ подгруппа групшы $G$, то пусть

$$
H_{G}=\bigcap_{g \in G} H^{g}
$$

Очевидно, что $H_{G}$ - наибольшая нормальная подгруппа групшы $G$, лежашая в $H$. Подгруппу $H$ будем назьвать $\varphi$-допустимой, если

$$
(N \cap H)^{\varphi} \subseteq N^{\varphi} \cap H .
$$

Если группа $G$ не содержит собственных $\sigma$-инвариантных $\varphi$-допустимых нормальных подгрупп, то будем ее называть $(\sigma, \varphi)$-простой.

СлЕДСТВИЕ 3. Пусть $H-\sigma$-инвариантная нормальная подгруппа $(\sigma, \varphi)$-группь G. Факторгруппа $G / H$ обладает $(\sigma, \varphi)$-свойством тогда и только тогда, когда $H$ является $\varphi$-допустимой подгруппой в $G$. Ассоииированная с әруппой $G / H$ лупа $S_{1}=S_{1}(G / H)$ является гомоморфным образом $S=S(G)$, при әтом $S \simeq S_{1}$ тогда и только тогда, когда $N^{\varphi} \cap H \subseteq I_{N^{\varphi}}$. 
ДокАЗАТЕЛЬСТво. Положим $K=N \cap H$ и $M=N^{\varphi} \cap H$, и пусть $K^{\varphi} \subseteq M$. Замечая, что $N / K \simeq N^{\varphi} / K^{\varphi}$, видим, что гомоморфизм $G \rightarrow G / H$ индуцирует естественньй гомоморфизм $\varphi^{\prime}: N / K \rightarrow N^{\varphi} / M$ с ядром $M / K^{\varphi}$. Очевидно, что тогда групша $G / H$ будет $\left(\sigma, \varphi^{\prime}\right)$-группой. Обратно, существование гомоморфизма $\varphi^{\prime}$ требует, чтобы его ядро $\operatorname{Ker}(\varphi)=M / K^{\varphi}$. Отсюда следует, что $K^{\varphi} \subseteq M$. Для построения гомоморфизма $S \rightarrow S_{1}$ можно использовать отображение $P_{x} \rightarrow P_{x} M$. В этом случае очевидно, что $S \simeq S_{1}$ тогда и только тогда, когда элементы $M$ действуют тривиально на $S$, или, эквивалентно, на смежных классах по $I$. Утверждение доказано.

3. Пусть $S$ - лупа Бола. Обозначим через $\widetilde{G}(S)$ групу, заданную порождаюшими $P_{x}$ и $R_{x}$, где $x \in S$, и определяюшими соотношениями $(7)-(10)$. Очевидно, что $\widetilde{G}(S)$ допускает инволютивный автоморфизм $\sigma$, определяемый отображениями $P_{x} \rightarrow R_{x}^{-1}$ и $R_{x} \rightarrow P_{x}^{-1}$. Группу $\widetilde{G}(S)$ можно представить в виде свободного произведения

$$
\widetilde{G}(S)=\left(A * B ; A_{1}=B_{1}, \psi\right)
$$

групп

$$
A=\left\langle P_{x} \mid x \in S\right\rangle \quad \text { и } \quad B=\left\langle R_{x} \mid x \in S\right\rangle
$$

с подгруппами

$$
A_{1}=\left\langle P_{x} P_{y} P_{x y}^{-1} \mid x, y \in S\right\rangle \quad \text { и } \quad B_{1}=\left\langle R_{x}^{-1} R_{y}^{-1} R_{x y} \mid x, y \in S\right\rangle,
$$

объединенными в соответствии с изоморфизмом $\psi: A \rightarrow B$. В этом случае автоморфизм $\sigma$ групп $\widetilde{G}(S)$ можно определить, полагая $\sigma=\psi$ на $A$ и $\sigma=\psi^{-1}$ на $B$. При этом подгрупша $\tilde{I}$, порожденная в $\widetilde{G}(S)$ всеми элементами вида $P_{x} P_{y} P_{x y}^{-1}$, будет совпадать с множеством всех неподвижных точек автоморфизма $\sigma$.

Tеорема 2. Группа $\widetilde{G}(S)$ является $(\sigma, \varphi)$-группой и $S(\widetilde{G}(S))=S$. Всякая другая $(\sigma, \varphi)$-группа $G$ такая, что $S(G)=S$, является гомоморфным образом группь $\widetilde{G}(S)$.

ДокАЗАТЕЛЬСтво. Пусть $\widetilde{N}$ - подгруппа в $\widetilde{G}=\widetilde{G}(S)$, порожденная всеми $L_{x}=$ $P_{x}^{-1} R_{x}^{-1}$, где $x \in S$. Перепишем соотношение (10) в виде

$$
R_{y} L_{x} R_{y}^{-1}=L_{y}^{-1} L_{x y} \quad \text { и } \quad P_{y}^{-1} L_{x} P_{y}=L_{x y} L_{y}^{-1}
$$

и заметим, что $L_{x}^{\sigma}=L_{x}^{-1}$. Очевидно, что подгруппа $\widetilde{N}$ является $\sigma$-инвариантным нормальным делителем грушы $\widetilde{G}$. Пусть $W$ - произвольньй элемент групшы $\widetilde{G}$, представленный в виде слова от порождающих $P_{x}$ и $R_{x}$, где $x \in S$. Тогда

$$
\left(W P_{x}\right)^{-1}\left(W P_{x}\right)^{\sigma}=P_{x}^{-1} W^{-1} W^{\sigma} P_{x} L_{x}, \quad\left(W R_{x}\right)^{-1}\left(W R_{x}\right)^{\sigma}=R_{x}^{-1} W^{-1} W^{\sigma} R_{x} L_{x^{-1}}^{-1} .
$$

Используя индукцию по длине слова $W$, доказываем, что $\widetilde{N}=\left\langle g^{-1} g^{\sigma} \mid g \in \widetilde{G}\right\rangle$. Кроме того,

$$
A_{1} P_{x} P_{y} W=A_{1} P_{x y} W
$$

для любого $W \in A$. Снова используя индукцию по длине слова доказываем, что каждый правый смежный класс группы $A$ по подгруппе $A_{1}$ содержит элемент $P_{x}$ для некоторого $x \in S$. 
Теперь рассмотрим гомоморфизм групшы $\widetilde{G}$ на ее подгрупшу $A$, которьй действует тождественно на $A$ и переводит элемент $b \in B$ в элемент $b^{\sigma}$. Легко видеть, что подгруппа $\widetilde{N}$ совпадает с ядром этого гомоморфизма и потому тривиально пересекается с подгруппой $A$. Аналогично, пересечение подгруппы $\widetilde{N}$ с подгруппой $B$ тривиально, и потому $\widetilde{N}$ - свободная группа (см. [3]). С другой стороны, существует гомоморфизм группы $A$ на группу правых умножений лупы $S$ такой, что каждый порождающий $P_{x}$ переходит в оператор правого умножения на элемент $x$ в $S$. Поэтому $P_{x} \in A_{1}$, только если $x=e$, и, следовательно, $P_{x} P_{y} \in A_{1}$, только если $y=x^{-1}$. Поэтому подгрупша $\tilde{N}$ свободно порождается элементами $L_{x}$, где $x \in S$ и $x \neq e$. Таким образом, существует гомоморфизм $\varphi$ группы $\widetilde{N}$ на $A$ такой, что $\varphi\left(L_{x}\right)=P_{x}$ для всех $x \in S$. Отсюда следует, что $\widetilde{G}$ является $(\sigma, \varphi)$-групшой. Из теоремы 1 следует тогда, что $S(\widetilde{G}(S))=S$.

Далее, пусть $G$ - другая $(\sigma, \varphi)$-группа такая, что $S(G)=S$. Тогда в силу следствия 1 к теореме 1 группа $G$ обладает системой порождающих $P_{x}$ и $R_{x}, x \in S$, удовлетворяющих соотношениям (7)-(10), и потому является гомоморфным образом группы $\widetilde{G}$. Теорема доказана.

СлЕДСТВИЕ 1. Для любой лупы Бола $S$ существует единственная, с точностью до изоморфизма, ассоциированная с ней группа $G_{0}=G_{0}(S)$ такая, что всякая другая группа $G$, ассоииированная $c S$, имеет $G_{0}$ своим гомоморфным образом. $\Pi p u$ əтом $\left(I_{0}\right)_{N_{0}^{\varphi}}=1$.

ДокАЗАТЕЛЬСтво. Пусть $H_{1}$ и $H_{2}$ - две максимальные нормальные $(\sigma, \varphi)$-подгруппы в $\widetilde{G}=\widetilde{G}(S)$ такие, что $\widetilde{N}^{\varphi} \cap H_{i} \subseteq \tilde{I}_{\tilde{N}^{\varphi}}$. Очевидно, что пересечение

$$
\tilde{N} \cap\left(H_{1} H_{2}\right)=\left(\tilde{N} \cap H_{1}\right)\left(\tilde{N} \cap H_{2}\right)
$$

Следовательно, $\sigma$-инвариантная нормальная подгруппа $H=H_{1} H_{2}$ также является $\varphi$-допустимой, и поэтому $H_{1}=H_{2}=H$. Пусть $G_{0}=\widetilde{G} / H$. Если $G$-любая другая группа, ассоциированная с $S$, то $G=\widetilde{G} / K$ для некоторой нормальной $(\sigma, \varphi)$-подгрупшы $K$ в $\widetilde{G}$. С другой стороны, следствие 3 к теореме 1 утверждает, что пересечение $\widetilde{N} \varphi K \subseteq$ $\tilde{I}_{\tilde{N} \varphi}$. Поэтому $K \subseteq H$ и $G_{0} \simeq G /(H / K)$.

Далее, группа $\widetilde{G}=\left\langle x, x^{\sigma} \mid x \in \widetilde{N}^{\varphi}\right\rangle$ и пересечение $\tilde{I}^{x} \cap \tilde{I}=\tilde{I}^{x^{\sigma}} \cap \tilde{I}$. Поэтому подгруппа $(\tilde{I})_{\tilde{N} \varphi}$ является нормальной $(\sigma, \varphi)$-подгрупшой в $\widetilde{G}$. Отсюда следует, что $\left(I_{0}\right)_{N_{0}^{\varphi}}=1$.

СлЕДСТВИЕ 2. Лупа Бола $S$ является простой тогда и только тогда, когда ассочиированная с ней группа $G_{0}(S)$ является $(\sigma, \varphi)$-простой и хопфовой. При әтом подаруппа $N_{0}^{\varphi}$ в $G_{0}(S)$ является простой группой.

ДокАЗАТЕЛЬСтво. По следствию 1 к теореме 2 подгруппа $\left(I_{0}\right)_{N_{0}^{\varphi}}=1$. Поэтому если $H$ - собственная нормальная $(\sigma, \varphi)$-подгруппа групшы $G_{0}$, то $G_{0}(S) / H$ - группа с $(\sigma, \varphi)$-свойством, ассоциированная лупа которой является собственным гомоморфньп образом лупы $S$. Обратно, если $S_{1}-$ собственная нормальная подлупа лупы $S$, то гомоморфизм $\widetilde{G}(S) \rightarrow \widetilde{G}\left(S / S_{1}\right)$ индуцирует гомоморфизм $G_{0}(S) \rightarrow G_{0}\left(S / S_{1}\right)$ с $\sigma$-инвариантным и $\varphi$-допустимым ядром; так что группа $G_{0}$ не $(\sigma, \varphi)$-проста.

Далее, пусть $K$ - нормальньй делитель грушы $N_{0}^{\varphi}$ и $\widetilde{K}-$ его прообраз в $\widetilde{N}^{\varphi}$. Очевидно, что гомоморфизм $\widetilde{N}^{\varphi} \rightarrow \widetilde{N}^{\varphi} / \widetilde{K}$ индуцирует гомоморфизм $S \rightarrow S / S_{1}$ с ядром $S_{1}=e \widetilde{K}$, где $e-$ единища лупы $S$. Если $S$ - простая лупа, то $e g=e$ для всех $g \in \widetilde{K}$. Поэтому следствие 2 к теореме 1 требует, чтобы $\widetilde{K} \subseteq \tilde{I}$. Используя такие же рассуждения, 
что и в следствии 1 к теореме 2 , показываем, что $\widetilde{K}$ - нормальньй $\varphi$-допустимый делитель группы $\widetilde{G}(S)$. Следовательно, $\widetilde{K} \widetilde{K}^{\sigma}-$ нормальная $(\sigma, \varphi)$-подгрупша групшы $\widetilde{G}(S)$. Отсюда следует, что $K=1$.

СлЕдСТВИЕ 3. Если $S$ - простая лупа Бола и nодгруппа группы $G_{0}=G_{0}(S)$, то $H=N_{0}$ uлu $H \times H^{\sigma}=G_{0}$.

ДокАЗАТЕльство. Пусть $H$ - собственная нормальная $\sigma$-инвариантная подгруппа группы $G_{0}$. Тогда пересечение $H \cap N_{0} \neq 1$. Иначе $H$ будет $\varphi$-допустимой подгруппой $G_{0}$, что невозможно в силу следствия 2 к теореме 2 . Пусть $K=H \cap N_{0}$. Тогда $K-$ собственная нормальная $\sigma$-инвариантная подгрупша групшы $G_{0}$. Если $K \subseteq \operatorname{Ker}(\varphi)$, то $K$ является $\varphi$-допустимой подгруппой в $G_{0}$, что невозможно. Если $N_{0} \neq K \nsubseteq \operatorname{Ker}(\varphi)$, то $K^{\varphi}$ - собственная нормальная подгруппа в $N_{0}^{\varphi}$, что невозможно в силу простоты $N_{0}^{\varphi}$. Следовательно, $K=N_{0}$ и поэтому $N_{0} \subseteq H$. С другой стороны,

$$
N_{0} / H \simeq\left(G_{0} / N_{0}\right) /\left(H / N_{0}\right)=N_{0}^{\varphi} /\left(H / N_{0}\right) .
$$

Отсюда следует, что $H=N_{0}$.

Теперь пусть $H$ - собственная нормальная подгруппа $G_{0}$, и пусть $H \neq N_{0}$. Тогда очевидно, что $H \cap H^{\sigma} \neq N_{0}$ и $H H^{\sigma} \neq N_{0}$. Поэтому $H \cap H^{\sigma}=1$ и $H H^{\sigma}=G_{0}$. Иначе, $G_{0}=H \times H^{\sigma}$.

СлЕДСТВИЕ 4. Если $S$ - простая лупа Бола и $H$ - нормальная подгруппа группь $G_{0}=G_{0}(S)$, то справедливо одно из следующих утверждении:

a) $G_{0}$ - простая группа и $S$ - лупа Муфанг;

b) $H=N_{0} u G_{0}=N_{0} \lambda N_{0}^{\varphi}$ - полупрямое произведение подгрупп $N_{0} u N_{0}^{\varphi}$;

c) $H \neq N_{0}$, әруппа $G_{0}=H \times H^{\sigma}$ u $S$ - лупа Муфанг.

ДокАЗАТЕльство. Если $G_{0}$ - простая групп, то $N_{0}=G_{0}$ и, следовательно, подгруппа $\operatorname{Ker}(\varphi)$ в $N_{0}$ будет нормальной подгруппой групшы $G_{0}$. Очевидно, что $\operatorname{Ker}(\varphi) \neq$ $G_{0}$. Поэтому $\operatorname{Ker}(\varphi)=1$. Используя теорему 1 , доказьваем, что $S$ - лупа Муфанг.

Пусть $H-\sigma$-инвариантная собственная нормальная подгруппа группы $G_{0}$. Тогда, как и в предыдущем следствии, $H=N_{0}$. С одной стороны, очевидно, что группа $G_{0}$ порождается подгруппами $N_{0}$ и $N_{0}^{\varphi}$. С другой стороны, следствие 2 к теореме 2 утверждает, что $N_{0}^{\varphi}-$ простая групша и, следовательно, пересечение $N_{0} \cap N_{0}^{\varphi}=1$. Поэтому группа $G_{0}$ является полупрямым произведением подгрупп $N_{0}$ и $N_{0}^{\varphi}$.

Пусть $H$ - собственная нормальная подгруппа группы $G_{0}$ и пусть $H \neq N_{0}$. Тогда, как и в предыдущем следствии, группа $G_{0}=H \times H^{\sigma}$. Пусть $H \cap N_{0}=M \neq 1$. Очевидно, что $M$ - собственная нормальная подгруппа группы $G_{0}$. С другой стороны, пересечение $M \cap M^{\sigma}=1$, и потому $M \neq N_{0}$. Но тогда $M \times M^{\sigma}=G_{0}$ и, следовательно, $M=H$. Таким образом, $N_{0}=G_{0}$. Отсюда следует, что подгруппа $\operatorname{Ker}(\varphi)$ в $N_{0}$ будет нормальной подгруппой групшы $G_{0}$. Пусть $\widetilde{K}-$ ее прообраз в $\widetilde{G}=\widetilde{G}(S)$. Вьше (см. доказательство теоремы 2) показано, что факторгруппа $\widetilde{N} / \widetilde{K} \simeq \widetilde{G} / \widetilde{N}$. Поскольку группы $\widetilde{G}$ и $\widetilde{N}$ являются $\sigma$-инвариантными, то $\sigma$-инвариантными будут группа $\widetilde{K}$ и, следовательно, ее образ в группе $G_{0}$. Таким образом, $\operatorname{Ker}(\varphi)=1$. Но тогда $N_{0}^{\varphi}=H \times H^{\sigma}$, что невозможно в силу простоты группы $N_{0}^{\varphi}$. Поэтому $H \cap N_{0}=1$ и, следовательно, $H \times N_{0}-$ нормальная подгрупша группы $G_{0}$. Очевидно, что $H \times N_{0}$ не может быть собственной нормальной подгруппой группы $G_{0}$. Поэтому $H \times N_{0}=G_{0}$ и, следовательно, $H \simeq N_{0}$ и $G_{0} / N_{0} \simeq N_{0}$. С другой стороны, $G_{0}=N_{0} \lambda N_{0}^{\varphi}$ и, следовательно, $G_{0} / N_{0} \simeq N_{0}^{\varphi}$. Таким образом, $N_{0}^{\varphi} \simeq N_{0}$, ядро $\operatorname{Ker}(\varphi)=1$ и $S$ - лупа Муфанг. 
4. Напомним (см. [4]), что группа $G$ назьвается финитно аппроксимируемой, если пересечение всех ее нормальных подгрупп конечного индекса есть единичная група. Группа $G$ финитно апшроксимируема тогда и только тогда, когда для каждого ее элемента $g \neq 1$ существует гомоморфизм $G$ в конечную групп, при котором $g$ не отображается в единицу. Известно (см. [3], [5]), в частности, что всякое свободное произведение двух конечных групп с объединенной подгруппой является финитно аппроксимируемой группой.

ТЕорема 3. Если $S$ - конечная лупа Бола порядка $n$, то $\widetilde{G}(S)$ - финитно аппроксимируемая группа, а $\widetilde{N}^{\varphi}-$ конечная группа порядка $<\frac{1}{2}$ en!, әде е - основание натурального логарифма.

ДОКАЗАТЕЛЬСТВо. Пусть $\Sigma=\langle\sigma\rangle$ и $\Sigma^{\prime}=\left\langle\sigma^{\prime}\right\rangle$, где $\sigma^{\prime}$ - инволютивньй автоморфизм группы $\tilde{N}^{\varphi}$, индуцируемый автоморфизмом $x \rightarrow x^{-1}$ симметрического пространства $S$. Тогда гомоморфизм $\widetilde{N} \rightarrow \widetilde{N}^{\varphi}$ индуцирует гомоморфизм $\varphi^{\prime}: \widetilde{N} \Sigma \rightarrow \widetilde{N}^{\varphi} \Sigma^{\prime}$ соответствуюших полупрямых произведений. Очевидно, что такой гомоморфизм переводит порождаюшие $L_{x} \sigma$ группы $\widetilde{N} \Sigma$ в порождающие $P_{x} \sigma$ группы $\widetilde{N}^{\varphi} \Sigma^{\prime}$. Пусть $v \in \widetilde{N}^{\varphi} \Sigma^{\prime}$ и пусть $\left(L_{x} \sigma\right)^{u}$ - произвольный прообраз элемента $\left(P_{x} \sigma^{\prime}\right)^{v}$ в $\tilde{N} \Sigma$. Очевидно, что

$$
\varphi^{\prime}:\left(L_{x} \sigma\right)^{L_{y}} \rightarrow P_{\left(y^{-1} x\right) y^{-1} \sigma^{\prime}}
$$

Поэтому существует такое $z \in S$, что $\varphi^{\prime}:\left(L_{x} \sigma\right)^{v} \rightarrow P_{z} \sigma^{\prime}$. Следовательно, група $\tilde{N}^{\varphi} \Sigma^{\prime}$ порождается конечным множеством элементов, каждый из которых в силу тождеств (6)-(8) конечного порядка и имеет конечное число сопряженных.

Пусть $|S|=n$. Тогда $P_{x_{1}} \sigma^{\prime}, \ldots, P_{x_{n}} \sigma^{\prime}-$ множество порождающих группы $\tilde{N}^{\varphi} \Sigma^{\prime}$ и сопряженных к ним. Покажем, что всякий элемент из $\tilde{N}^{\varphi} \Sigma^{\prime}$ может быть записан в виде слова $W\left(P_{x_{1}} \sigma^{\prime}, \ldots, P_{x_{n}} \sigma^{\prime}\right)$ длины $\leqslant n$. Действительно, $\left(P_{x_{i}} \sigma^{\prime}\right)^{2}=1$. Поэтому

$$
W=\left(\ldots P_{x_{i}} \sigma^{\prime} W_{1} P_{x_{i}} \sigma^{\prime} \ldots\right)=\left(\ldots\left(P_{x_{i}} \sigma^{\prime}\right)^{-1} W_{1} P_{x_{i}} \sigma^{\prime} \ldots\right)
$$

Так как, сопрягая $P_{x_{j}} \sigma^{\prime}$ посредством $P_{x_{i}} \sigma^{\prime}$, мы снова получим один из $P_{x_{1}} \sigma^{\prime}, \ldots, P_{x_{n}} \sigma^{\prime}$, длину слова $W$ можно уменьшить. Через конечное число шагов мы получим представление $W$ в виде слова длины $\leqslant n$. Поскольку $\widetilde{N}^{\varphi}-$ подгруппа индекса 2 в $\widetilde{N}^{\varphi} \Sigma^{\prime}$, порядок

$$
\left|\widetilde{N}^{\varphi}\right| \leqslant \frac{1}{2} \sum_{k=1}^{n} \frac{n !}{(n-k) !}<\frac{1}{2} e n !
$$

Поскольку всякое свободное произведение двух конечных груп с объединенной подгруппой является финитно аппроксимируемой группой, утверждение доказано.

СлЕДСТВИЕ. Простая лупа Бола $S$ является конечной тогда и только тогда, когда конечной является группа $G_{0}(S)$.

ДоКАЗАТЕЛЬСТво. Часть “только тогда” следует из теоремы 1 и определения группы $G_{0}(S)$. Докажем часть “тогда” нашего утверждения. Пусть $S$ - конечная лупа Бола и пусть

$$
\widetilde{G}(S)=\left(A * B ; A_{1}=B_{1}, \psi\right)
$$


- определенное выше свободное произведение групп с объединенной подгруппой. Положим $A_{2}=\left(A_{1}\right)_{A}$ и $B_{2}=\left(B_{1}\right)_{B}$. Естественные отображения групп $A$ и $B$ на фактор-группы $A / A_{2}$ и $B / B_{2}$ продолжаемы до гомоморфизма группы $\widetilde{G}(S)$ на групу

$$
G=\left(A / A_{2} * B / B_{2} ; A_{1} / A_{2}=B_{1} / B_{2}, \psi^{\prime}\right) .
$$

Очевидно, что группа $G$ ассоциирована с лупой $S$ и обладает $(\sigma, \varphi)$-свойством. Из теоремы 3 следует, что группы $A$ и $B$, а значит, и их факторгрупшы, конечны. Поэтому группа $G$ является финитно аппроксимируемой. Выберем элемент $g^{\prime} \in N \backslash \operatorname{Ker}(\varphi)$. Тогда существует нормальная подгруппа $K$ конечного индекса группы $G$ такая, что $g^{\prime} \notin K$. Пусть $M=(K \cap N)(K \cap N)^{\sigma}$. В силу следствия 1 к теореме 2 факторгруппа $A / A_{2}$ изоморфна подгруппе $N_{0}^{\varphi}$ группы $G_{0}(S)$. В силу следствия 2 к теореме $2 A / A_{2}$ - простая группа. Следовательно, $M \subseteq \operatorname{Ker}(\varphi)$ и $M$ - нормальный $(\sigma, \varphi)$-допустимьй делитель группы $G$. С другой стороны, $M$ - подгрупша конечного индекса в $N$, а $N$ - подгруппа конечного индекса в $G$. Следовательно, $M$ - подгруппа конечного индекса в $G$ и $G / M$ - конечная группа, обладающая $(\sigma, \varphi)$-свойством. Но тогда и группа $G_{0}(S)$, являющаяся в силу следствия 1 к теореме 2 гомоморфным образом $G / M$, также будет конечной группой.

\section{СПИСОК ЦИТИРОВАННОЙ ЛИТЕРАТУРЫ}

[1] Белоусов В. Д. Основы теории квазигрупп и луп. М.: Наука, 1967.

[2] Лоос О. Симметрические пространства. М.: Наука, 1985.

[3] Neumann B. H. An essay of free products of groups with amalgamations // Philos. Trans. Roy. Soc. London. Ser. A. 1954. V. 246. P. 503-554.

[4] Магнус В., Каррас А., Солитэр Д. Комбинаторная теория групп. М.: Наука, 1974.

[5] Baumslag G. On the residual finiteness of generalized free products of nilpotent groups // Trans. Amer. Math. Soc. 1963. V. 106. P. 193-209. 